\title{
HOMOLOGOUS RNA RECOMBINATION ALLOWS EFFICIENT INTRODUCTION OF SITE-SPECIFIC MUTATIONS INTO THE GENOME OF CORONAVIRUS MHV-A59 VIA SYNTHETIC CO-REPLICATING RNAS
}

\author{
Robbert van der Most, Leo Heijnen, Willy Spaan and Raoul de Groot \\ Department of Virology, Institute of Medical Microbiology \\ Faculty of Medicine, Leiden University \\ Postbus 320, 2300 AH Leiden, The Netherlands
}

\begin{abstract}
We describe a novel strategy to site-specifically mutagenize the genome of an RNA virus by exploiting homologous RNA recombination between synthetic defective interfering (DI) RNA and the viral RNA. Marker mutations introduced in the DI RNA were replaced by the wild-type residues during replication. More importantly, however, these genetic markers were introduced into the viral genome: even in the absence of positive selection MHV recombinants could be isolated. This finding provides new prospects for the study of coronavirus replication using recombinant DNA techniques. As a first application, we describe the rescue of the temperature sensitive mutant MHV Albany-4 using DI-directed mutagenesis. Possibilities and limitations of this strategy are discussed.
\end{abstract}

\section{INTRODUCTION}

During mixed infection with different MHV strains, RNA recombination occurs at a remarkably high frequency, both in tissue culture and in infected mice (1-5). Although the mechanism is still unknown, homologous RNA recombination in coronavirus-infected cells presumably occurs via template switching ('copy-choice'). As proposed for picornavirus RNA recombination $(6,7)$, polymerase complexes containing nascent RNA are thought to dissociate from their original template and anneal to another, after which RNA synthesis proceeds (2).

We are interested in genetic manipulation of coronaviruses. For several other RNA viruses full-length cDNA clones have been constructed from which infectious RNA can be transcribed in vitro (8-11). However, the construction of a full-length cDNA clone of a 
coronavirus has been hampered by the extreme length of the coronavirus genome. Here, we describe an alternative strategy to site-specifically introduce mutations into the MHV genome. This strategy exploits the high-frequency RNA recombination that occurs in MHVinfected cells $(1,2)$ and involves the use of synthetic defective interfering (DI) RNAs. We provide evidence for homologous RNA recombination between DI RNA and the standard virus genome. Marker mutations introduced into the synthetic DI RNA were replaced by the wild-type residues during replication in MHV-infected cells. More importantly however, these marker mutations were incorporated into the genome of MHV-A59. DI-directed mutagenesis provides exciting new prospects for molecular genetic studies on coronaviruses, as was demonstrated by the rescue of the temperature-sensitive mutant Albany-4. This work was previously published in Nucleic Acids Research (vol. 20, p.3375-3388, 1992).

\section{RESULTS}

\section{Construction of pMIDI-C}

We previously reported the construction of pMIDI, a full-length cDNA clone of an MHV DI RNA (12). pMIDI consists of three non-contiguous regions of the MHV genome, namely the 5' most 3889 nucleotides, the 3 ' most 806 nucleotides and in between, 799 nucleotides derived from ORF1b of the polymerase gene. The three segments are joined inframe, although in pMIDI the reading frame is interrupted by a UAA termination codon at position 3357 (12). To study recombination between MHV DI RNAs and the MHV genome, we constructed a pMIDI-derivative, pMIDI-C, in which we introduced three silent point mutations at positions 1778, 2297, 3572 (mutations A, B and C, respectively; Fig. 1). Marker mutations $A(G \rightarrow A)$ and $B(T+C)$ were introduced in pMIDI by PCR-mutagenesis (13). The termination codon at position 3357 was replaced by the wild-type CAA codon by exchanging the XhoII-SpeI fragment (nucleotides 3237-3689) of pMIDI for the corresponding fragment derived from an independent, DI-derived cDNA clone, pDI02 (12). This fragment contained the wild-type CAA codon at position 3357 and a serendipitous $\mathrm{T} \rightarrow \mathrm{C}$ mutation at position 3572 (Marker C), presumably acquired during cDNA synthesis.

\section{Point mutations in MIDI-C are replaced by the wild-type sequence during replication in MHV-A59 infected cells}

RNA transcribed from pMIDI-C was used for transfection of MHV-infected mouse L-cells as described previously (12). At $12 \mathrm{hrs}$ after transfection the tissue-culture supernatant (passage 0 virus), containing virus and DI particles, was harvested and passaged twice on fresh L-cells, yielding passage 1 and 2 virus. Total intracellular RNA was isolated from passages 0,1 and 2 and analyzed by hybridization using (-)-sense oligonucleotide probe VI (Fig. 1). MIDI-C RNA was replicated in MHV-infected cells and strongly interfered with viral mRNA synthesis in cells infected with passage $1(\mathrm{p} 1)$ virus/DI mixture (not shown). To determine whether recombination had occurred between the MHV genome and the synthetic DI RNA, the sequence of p2 MIDI-C RNA was examined. For this purpose, the DI RNA was subjected to cDNA synthesis followed by PCR amplification (RT-PCR) using the oligonucleotide primers I and III (Fig. 1). To prevent in vitro recombination of genomic and DI sequences during RT-PCR, the MIDI-C RNA used as a template was gel-purified. DI-specific PCR-DNA was digested with HindIII (positions 1985 and 3782 in pMIDI-C) and cloned into pUC20. Sequence analysis of 64 independent clones 
showed that mutations B and C had been replaced by the wild-type sequence in 14 (22\%) and 4 clones $(6 \%)$ respectively.

Since (i) the mutations were exclusively replaced by the wild-type sequence and (ii) the frequency with which the mutations were replaced depended on their distance from the artificial junction of the $1 \mathrm{a}$ and $1 \mathrm{~b}$ segment, reversion by point mutation seems very unlikely. In fact, these results are best explained by homologous RNA recombination between MIDI-C RNA and the genome of the standard virus.

\section{Isolation of MHV recombinants}

RNA recombination between MIDI-C RNA and the viral genome could, in principle, also yield recombinant viruses carrying the marker mutations. To study this possibility, p2 virus was plaqued and 150 well-isolated plaques were used to inoculate mouse L-cells. Total cellular RNA, isolated from the infected cells, was subjected to RT-PCR using oligonucleotides I and II (Fig. 1). In all cases a DNA fragment with the expected length of $0.6 \mathrm{~kb}$ was generated. Evidently, the $0.6 \mathrm{~kb}$ fragment would also be produced with MIDI-C

POLYMERASE GENE

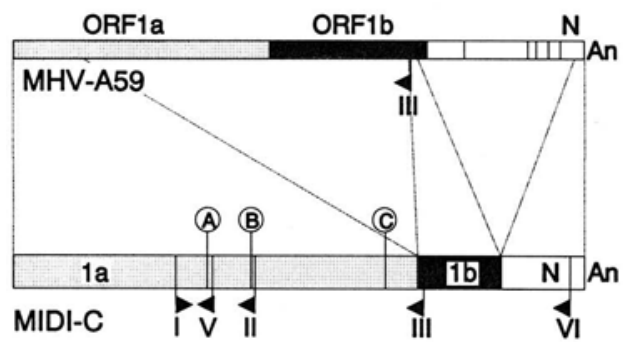

ACGU

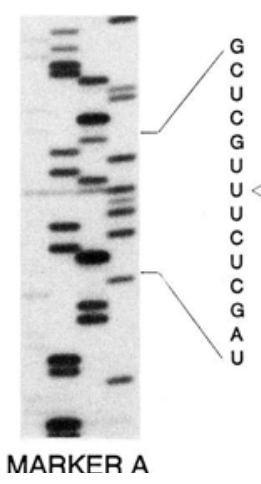

$A C G U$

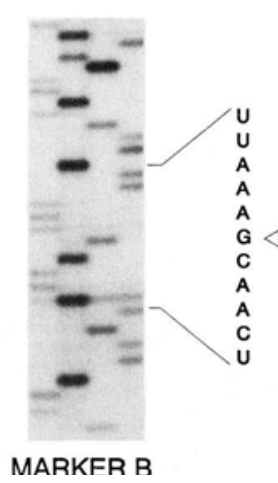

Higure 1. Schematic representation of the structure of MIDI-C RNA and the MHV-A59 genome. The different parts of MIDI-C, derived from the 5'end (ORF1a), ORF1b and $3^{\prime}$ end of the MHV-A59 genome $(N)$ are indicated. The locations are shown of marker mutations A, B and C, and of the oligonucleotides I, II, III, V and VI. The orientations of these oligonucleotides are indicated by arrowheads.

Figure 2. Sequence analysis of RNA isolated from cells infected with MHV recombinant 4, according to Fichot and Girard (15). Mutations A and B were analyzed using oligonucleotides $\mathrm{V}$ and II, respectively. Sequences are presented as $(-)$ strand RNA. Arrowheads indicate the introduced mutations. Identical results were obtained for recombinant 138 (not shown).

RNA as template (Fig. 1). However, MHV DIs are rapidly lost by infecting at a low m.o.i. $(12,14)$ and the obtained PCR fragments were therefore expected to be genome-specific.

The PCR-DNAs were screened for the presence of marker mutation B by differential hybridization using the 15-mer oligonucleotide probe IV (5' TGTCAACGAAATTCT 3', the guanine residue complementary to the introduced cytidine is underlined). PCR-DNA derived from two plaques, 4 and 138, specifically hybridized with this probe (not shown). The RNA preparations that had been used for RT-PCR were confirmed to be devoid of MIDI-C RNA by Northern blot analysis (not shown). 
To determine whether viruses 4 and 138 were true recombinants, four consecutive plaque purifications were performed. Of each plaque generation, three to five well-isolated plaques were analyzed by differential hybridization of PCR-amplified cDNA. Wild-type MHV, treated identically, served as a negative control. In all cases, the progeny of viruses 4 and 138 contained mutation B (not shown), confirming that this genetic marker had been introduced into the viral genome and strongly arguing against MIDI-C contamination.

As a final control, we performed direct RNA sequencing. Stocks of viruses 4 and 138, that had been plaque-purified twice, were used to infect L-cells at an m.o.i. of 10 . Intracellular RNAs were harvested and used for RNA sequence analysis using the oligonucleotide primers II and V (Figs. 1, 2). In addition to mutation B, the RNAs of viruses 4 and 138 contained mutation A (Fig. 2). Analysis of virus 4 RNA showed that mutation $\mathrm{C}$ was absent (not shown).

On the basis of our combined data, we concluded viruses 4 and 138 to be MHV mutants, generated by homologous RNA recombination between the synthetic MIDI-C RNA and the MHV-A59 genome.

\section{Rescue of the MHV Albany- 4 ts-mutant by homologous recombination}

Having demonstrated that sequences of synthetic DI RNAs can be introduced into the viral genome via RNA recombination, we explored 'DI-directed mutagenesis' as a means to identify and localize mutations that result in a conditionally lethal phenotype. For this purpose, we used MHV strain Albany-4, a temperature-sensitive (ts-) mutant of MHVA59. The ts-phenotype of this virus is the result of an 87 nucleotides deletion in the nucleocapsid (N) gene (nt $1138-1224$ of the $\mathrm{N}-\mathrm{ORF}$ ). At $39^{\circ} \mathrm{C}$, virus growth is impaired. Also, incubation of Albany-4 virions at $39^{\circ} \mathrm{C}$ abolishes infectivity (16).

pMIDI-C contains the $3^{\prime}$ terminal 510 nucleotides of the wild-type N-ORF including the 87 nucleotides that are deleted in Albany-4. Incorporation of the N-sequences of MIDIC RNA into the Albany-4 genome via RNA recombination should eliminate the ts-defect and generate wild-type MHV. To distinguish rescued Albany-4 recombinants from MHVA59 contaminants, a silent $\mathrm{T} \rightarrow \mathrm{A}$ substitution was introduced into the nucleocapsid sequence of pMIDI-C (position 5030, corresponding to position 1200 of the N gene) by PCRmutagenesis, yielding plasmid pMIDI-C*.

In this set of experiments, we used DNA transfection and in vivo transcription by vaccinia virus-expressed $T 7$ polymerase as an alternative to RNA transfection to introduce MIDI-C* into MHV-infected cells. First, L-cells were infected with recombinant vaccinia virus vTF7-3 (17) at an m.o.i of 5 for 45 min, followed by DNA or mock-transfection at 90 min post infection. Two hours after transfection the cells were super-infected with Albany-4 at an m.o.i. of 10 . After a $25 \mathrm{hr}$ incubation at $33^{\circ} \mathrm{C}$, the tissue culture supernatants were harvested and passaged once at $33^{\circ} \mathrm{C}$ in fresh L-cells, followed by a single passage at $39^{\circ} \mathrm{C}$. Vaccinia virus remains cell-associated, and is therefore lost during passage. The $39^{\circ} \mathrm{C} / \mathrm{p} 2$ virus stocks were used to infect monolayers of L-cells at $37^{\circ} \mathrm{C}$. Intracellular RNAs were separated in formaldehyde-agarose gels and hybridized to 5 '-end labelled oligonucleotide VI. This probe binds to the sequence, that has been deleted in Albany-4 (Fig. 3) and is therefore specific for the wild-type N-gene. Strikingly, probe VI not only detected MIDI-C* RNA but also the nested set of MHV mRNAs (Fig. 3, lanes 'MOCK' and 'MIDI-C*'), indicating that during passage, viruses had accumulated that had incorporated MIDI-C* sequences into their genomes.

To obtain direct evidence for this, the $\mathrm{p} 2$ virus/DI mixture was plaqued at $39^{\circ} \mathrm{C}$ and virus from 4 plaques was propagated in L-cell monolayers. After hybridization of the intracellular RNAs to oligonucleotide VI, the nested set of MHV RNAs was found; MIDI- 
C* RNA was not detected (Fig. 3, lanes 1-4). Sequence analysis of these RNA preparations showed that the wild-type $\mathrm{N}$-sequence had been restored and that the $\mathrm{U} \rightarrow \mathrm{A}$ marker mutation in the N-ORF was present (Fig. 4), providing formal evidence that the isolated viruses were generated by recombination between MIDI-C* RNA and the Albany-4 genomic RNA.

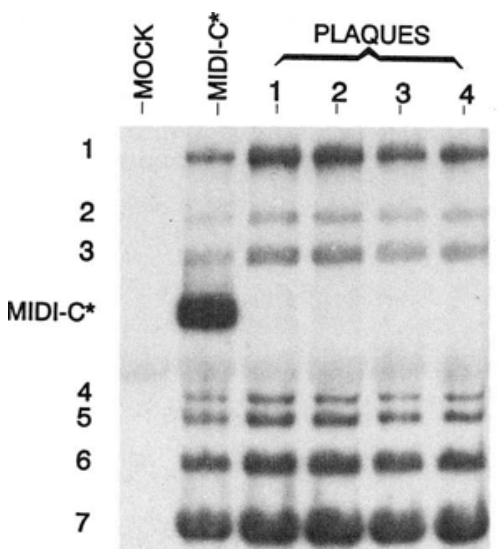

Fig. 3. Hybridization analysis of intracellular RNAs of MHV Albany-4 recombinants. Stocks of MHV Albany-4 (p2, grown at $\left.39^{\circ} \mathrm{C}\right)$ obtained after initial transfection with pMIDI-C* or mocktransfection were either used to infect fresh L-cells at $37^{\circ} \mathrm{C}$ to isolate intracellular RNAs (lanes 'Mock' and 'MIDI-C*') or plaqued at $39^{\circ} \mathrm{C}$. Virus from four randomly-chosen plaques was propagated and intracellular RNAs were isolated (lanes 1-4).

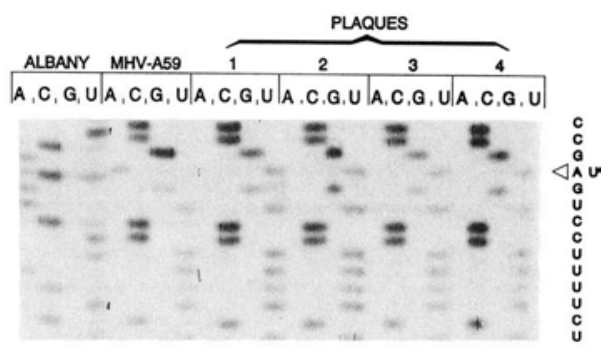

Fig. 4. RNA sequence analysis of recombinant Albany-4 viruses. Intracellular RNA of four plaque-purified recombinant Albany-4 viruses, described in Fig. 3, was subjected to sequence analysis. Intracellular RNA from Albany-4- and MHV-A59-infected cells served as controls. Sequences are presented as (-)strand RNA. An arrowhead indicates the introduced marker mutation.

\section{DISCUSSION}

For the coronavirus MHV homologous recombination of genomic RNAs has been well documented $(1-4,18)$. Here, we extend these observations by providing evidence for recombination between DI RNAs and the MHV-A59 genome. We have shown that silent mutations introduced into the ORF1a sequence of MIDI-C were exclusively replaced by the wild-type residues during DI replication in MHV-infected cells. These marker mutations, however, were not replaced at an equal rate: markers B and C, located 1500 and 300 nucleotides upstream of the artificial ORF1a/1b border in MIDI-C, were replaced in $22 \%$ and $6 \%$ of the passage 2 DI RNAs, respectively. Apparently, the frequency of replacement correlated with the distance between the mutation and the ORF1a/1b border, i.e. the region in which template-switching has to occur in order to remove the mutation while maintaining the original MIDI-C structure. These finding are in accordance with the current model for coronavirus RNA recombination.

Most convincingly, recombination between MIDI-C RNA and the MHV genomic RNA was demonstrated by the identification of viruses that had incorporated the genetic markers A and B into their genomes. It should be emphasized that these viruses were isolated in the absence of selection. These findings lead to the important conclusion that synthetic DI RNAs can be used to site-specifically alter the MHV genome by exploiting RNA recombination. The potential of DI-directed mutagenesis is illustrated by the rescue of the conditionally lethal mutant Albany-4. The ts-phenotype of this mutant is caused by a deletion at the $3^{\prime}$ end of the $\mathrm{N}$-gene (16). Viruses that had restored the $\mathrm{N}$-gene by 
recombination with MIDI-C* RNA, were selected for by passaging the virus/DI mixture at the restrictive temperature.

Clearly, there will be limitations in this system: thus far, we have introduced mutations only in the $5^{\prime}$ and $3^{\prime}$ terminal regions of the MHV genome. It remains to be determined whether mutations can be introduced efficiently into more internal regions, since this would require double recombination events. Also, in the case of mutations causing reduced replication in vitro, the screening for recombinant viruses will be difficult. Presumably, such problems can be solved by improving screening procedures and by applying selection, e.g. via rescue of ts-markers (this paper) or by using neutralizing antibodies (18). Studies to address these issues are currently in progress.

Recently, a publication by Koetzner et al. (19) appeared describing the rescue of MHV Albany- 4 by targeted RNA recombination using a synthetic mRNA 7 homologue. However, the observed recombination frequency using this method was considered too low to allow direct identification of recombinants without selection. In fact, it was anticipated that a more general applicability of targeted RNA recombination would require finding conditions that favour higher rates of recombination of exogenous RNA. The results described here show that by using synthetic co-replicating DI RNAs site specific mutations can be introduced efficiently into the MHV genome.

\section{ACKNOWLEDGMENTS}

We thank Dr. Paul Masters for generously providing MHV Albany-4 and Dr. Willem Luytjes for stimulating discussions. R.G.M. was supported by grant 331-020 from the Dutch Foundation for Chemical Research (SON). We gratefully acknowledge Oxford University Press for permission for the use of copyright material.

\section{REFERENCES}

1. M.M.C. Lai, R.S. Baric, S. Makino, J.G. Keck, J. Egbert, J.L. Leibowitz and S.A. Stohlman, J. Virol., 56:449-456 (1985).

2. S. Makino, J.G. Keck, S.A. Stohlman and M.M.C. Lai, J. Virol. 57:729-737 (1986).

3. J.G. Keck, S.A. Stohlman, L.H. Soe, S. Makino and M.M.C. Lai, Virology 156:331-341 (1987).

4. J.G. Keck, L.H. Soe, S. Makino, S.A. Stohlman and M.M.C. Lai, J. Virol. 62:1989-1998 (1988).

5. J.G. Keck, G.K. Matsushima, S. Makino, J.O. Fleming, D.M. Vannier, S.A. Stohlman and M.M.C. Lai, J. Virol. 62:1810-1813 (1988).

6. K. Kirkegaard and D. Baltimore, Cell 47:433-443 (1986).

7. T.C. Jarvis and K. Kirkegaard, EMBO J. 11:3135-3145 (1992).

8. P. Ahlquist, R. French, M. Janda and L.S. Loesch-Fries, Proc.NathAcad.Sci. USA 81:7066-7070 (1984).

9. C.M. Rice, R. Levis, J.H. Strauss, H.V. and Huang, J. Virol. 61:3809-3819 (1987).

10. C.M. Rice, A. Grakoui, R. Galler and T.J. Chambers, New Biol. 1:285-296 (1989).

11. C.-J. Lai, B. Zhao, H. Hori and M. Bray, Proc.NatLAcad.Sci. USA 88:5139-5143 (1991).

12. R.G. van der Most, P.J. Bredenbeek and W.J.M. Spaan, J. Virol. 65:3219-3226 (1991).

13. J. Sambrook, E.F. Fritsch and T. Maniatis, Molecular Cloning. Cold Spring Harbor Laboratory Press, Cold Spring Harbor (1989).

14. S. Makino, F. Taguchi and K. Fujiwara, Virology 133:9-17 (1984).

15. O. Fichot and M. Girard, Nucleic Acids Res. 18:6162 (1990).

16. P.S. Masters and L.S. Sturman, Adv. Exp. Med. Biol. 276:235-238 (1990).

17. T.R. Fuerst, E.G. Niles, F.W. Studier and B. Moss, Proc.NathAcad.Sci. USA 83:8122-8126 (1986).

18. S. Makino, J.O. Fleming, J.G. Keck, S.A. Stohlman and M.M.C. Lai, Proc.NatlAcad.Sci. USA 84:6567-6571 (1987)

19. C.A. Koetzner, M.M. Parker, C.S. Ricard, L.S. Sturman and P.S. Masters, J. Virol. 66:1841-1848 (1992). 